\title{
A COMPARATIVE STUDY OF TENSION FREE MESH HERNIA REPAIR DONE UNDER LOCAL ANAESTHESIA VERSUS REGIONAL ANAESTHESIA
}

\author{
Vijay V. Kamat ${ }^{1}$, Aswathy Chandran ${ }^{2}$
}

${ }_{1}^{1}$ Associate Professor, Department of General Surgery, KIMS, Hubballi, Karnataka, India.

2Junior Resident, Department of General Surgery, KIMS, Hubballi, Karnataka, India.

\section{BACKGROUND}

ABSTRACT

Hernias are among the oldest known afflictions known to mankind and elective inguinal hernia repair is one of the most common operations performed by general surgeons. ${ }^{1}$ Two thirds of these are indirect and the remainder are direct inguinal hernias. Femoral hernias comprise only 3\% of all groin hernias. Men are 25 times more likely to have a groin hernia than women. An indirect inguinal hernia is the most common hernia, regardless of gender.1,2

\section{MATERIALS AND METHODS}

The study was a non-randomized controlled trial study conducted between October 2015 and November 2017 at a medical college hospital. Sample size was taken for convenience.

\section{RESULTS}

Both local \& spinal anaesthesia can be used for hernia repair on short stay bases, but spinal anaesthesia has higher complication rates compared to local anaesthesia. There is significant increase in general complications like hypotension, urinary retention, \& headache in spinal anaesthesia \& local complications like seroma, hematoma, scrotal oedema \& recurrence were similar in both groups.

\section{CONCLUSION}

Both local \& spinal anaesthesia can be used for hernia repair on short stay bases, but spinal anaesthesia has higher complication rates compared to local anaesthesia. Local anaesthesia is with less immediate post-operative complication, best suitable for short stay surgery when compared to spinal anaesthesia.

\section{KEYWORDS}

Hernioplasty, Hernia, Lichtenstein's Tension Free Hernioplasty

HOW TO CITE THIS ARTICLE: Kamat VV, Chandran A. A comparative study of tension free mesh hernia repair done under local anaesthesia versus regional anaesthesia. J. Evolution Med. Dent. Sci. 2019;8(11):764-768, DOI: 10.14260/jemds/2019/168

\section{BACKGROUND}

Hernias are among the oldest known afflictions to mankind and elective inguinal hernia repair is one of the most common operations performed by general surgeons. It is estimated that $5 \%$ of the population will develop an abdominal wall hernia, but the prevalence may be even higher. About $75 \%$ of all hernias occur in the inguinal region. ${ }^{3}$ Two thirds of these are indirect and the remainder are direct inguinal hernias. Femoral hernias comprise only $3 \%$ of all groin hernias. ${ }^{3,4}$ Men are 25 times more likely to have a groin hernia than women. An indirect inguinal hernia is the most common hernia, regardless of gender.5,6 The word "hernia" is a Latin term that means "rupture" of a portion of structure. This word in Greek means "bud".7 Inguinal hernia repair can be performed by using a variety of anaesthetic techniques such as, general anaesthesia, regional anaesthesia in the form of spinal or epidural anaesthesia, paravertebral block and local anaesthesia. 8,9

'Financial or Other Competing Interest': None.

Submission 17-01-2019, Peer Review 02-03-2019,

Acceptance 08-03-2019, Published 18-03-2019.

Corresponding Author:

Aswathy Chandran

Vysakham House, Ramakodam Lane,

Perumbhotta Road, Elamakkara,

Ernakulam-682026,

Kerala, India.

E-mail: aswathydot@gmail.com

DOI: $10.14260 /$ jemds $/ 2019 / 168$

\section{(c) (1) $(9)$}

The choice of anaesthesia and advantages of any one technique over another has been a subject of debate. Patient safety and the provision of optimum operating conditions for the surgeon are two main criteria for choice of anaesthetic technique for inguinal hernia repair. General and regional anaesthesia both have been reported to cause haemodynamic changes during induction and maintenance. Local anaesthesia provides increased safety for patients, better postoperative pain control, shorter recovery period with reduced duration of hospital stay and reduced cost, hence local anaesthesia is acceptable and safe technique for inguinal hernia surgery.

Local anaesthesia has been found to be the best anaesthesia for inguinal hernia repair. The use of local anaesthesia in hernia repair varies from a few per cent in Sweden, $18 \%$ in Denmark and up to almost 100\% in specialised institutions like Shouldice clinic, Toronto clinic and others totally dedicated to hernia surgery. However, general or spinal anaesthesia are still the most common types of anaesthesia being used in India and other developing countries. ${ }^{10,11}$

We plan to study the effectiveness of local anaesthesia in comparison to regional anaesthesia repairing uncomplicated inguinal hernia by evaluating post-operative pain and postoperative complications.

\section{MATERIALS AND METHODS}

The study was a non-randomized controlled trial study conducted between October 2015 and November 2017 at a medical college hospital. Patients attending the Medical 
College Hospital OPD and those who are admitted to the hospital for uncomplicated Inguinal Hernia Repair from October 2015 to November 2017 were included. They underwent a through clinical examination and the necessary pre-operative investigations. Surgical management based on clinical examinations and investigations. Based on the diagnosis, type of surgery was decided. Sample size was taken for convenience.

Post-operative recovery was accessed including complications like urinary retentions, postoperative pain, headache, seroma, haematoma, scrotal oedema, infection, recurrence and duration hospital stay. Post-operative pain will be evaluated using visual analogue scale at 30, 60120 \& 240 minutes. Further patients were followed up in surgery OPD for 6 months. Final outcome was evaluated. There were 30 cases of inguinal hernia repair done under local anaesthesia (Group A). And minimum of 30 cases of inguinal hernia repair done under spinal anaesthesia (Group B). Patients with recurrent hernia, Patients below 18, Patients with bilateral hernia, Patients with complicated hernias likeirreducibility, obstruction, strangulation, Patients with preoperative chronic pain problems, Patients with psychiatric problems and pregnancy and Patients with bleeding disorders and on anticoagulant treatment were excluded. Group A (Study Group) received a mixture of 40-45 $\mathrm{ml}$ of $1 \%$ lignocaine with adrenaline $1: 200000$ and $0.5 \%$ bupivacaine are used for local anaesthesia. Lignocaine is short acting and bupivacaine is a long acting agent. Ilioinguinal and Iliohypogastric nerve block with local infiltration anaesthesia is the method used for local anaesthesia and Group B (Control Group) received 3-4 ml of $0.5 \%$ Bupivacaine heavy is used for spinal anaesthesia (Done in L3-L4 space).

Chi square test and Independent student ' $\mathrm{t}$ ' test were used for analysis and analysis was done using SPSS version 20 (IBM). P value less than 0.05 was taken as significant.

\section{RESULTS}

The maximum number of patients are in the age group of 60 to 70 years and $30-40$ years. The mean age of the patients in GROUP A is 53.1 years whereas in GROUP B is 47.33 years. Majority of the study population had right sided inguinal hernia $(71.68 \%)$. The prevalence of indirect inguinal hernia was $91.66 \%$ and that of direct inguinal hernia was $8.33 \%$.

A total of $6.6 \%$ of the study population were diabetic and 8.3\% were hypertensive. $3.3 \%$ had cardiac illness and $1.6 \%$ had respiratory diseases. Hence, hypertension was the most common co-morbid condition observed among the study population. Tobacco chewing was the most common substance abuse observed among the study population. (28.33\%) followed by smoking (21.6\%) and alcoholism (18.33\%).

The mean duration of surgery in cases group A is $59.1+/-$ $8.76 \mathrm{~min}$ and in group B is $40.33 \pm 8.41 \mathrm{~min}$. The difference in the duration of surgery is statistically insignificant in both the groups with $\mathrm{p}$ value 1.61 .

A group 5 (16.67\%) patients experienced pain\& needed sedation \&analgesia (IV midazolam) during surgery, none of the patients experienced pain in group B. $(p=0.028$, significant).
None of the patients met with troublesome haemorrhage during surgery. Two (6.6\%) patients experienced hypotension in Spinal Anaesthesia group, who were treated with crystalloids \& vasopressors. No patient in Local Anaesthesia group experienced hypotension ( $\mathrm{p}=0.018$, significant). Two patients in Spinal Anaesthesia group (6.6\%) \& 2 patients $(6.6 \%)$ in Local Anaesthesia group experienced nausea \& vomiting. Seven patients in Spinal Anaesthesia group (23.3\%) experienced retention of urine which required catheterization ( $p=0.046$, significant).No patient with local anaesthesia experienced retention. Post-operative headache seen in $3(10 \%)$ patients in Spinal Anaesthesia group $(p=0.025$, significant). No patient with local anaesthesia experienced headache. One patient (3.3\%) patients in Spinal Anaesthesia group developed seroma. In total of 60 patients 1 (1.6\%) developed seroma. 0 (0\%) of SA group \& 1 (3.3\%) LA group developed scrotal oedema \& in total 1 (1.6\%) developed scrotal oedema. Hematoma developed in none of the patients. Wound infection accounts for 1 (1.60\%) which occurred in group B (Spinal Anaesthesia.).

The mean duration of post-operative hospital stay in group A is $2.6+/-0.81$ days and in group B is $3.81+/-1.62$ days. $40 \%$ of patients in both the groups stayed for a period of 2 days post operatively. The difference in duration of postoperative hospital stay is statistically significant in both the groups with $\mathrm{p}$ value 0.00032 .

On post-operative two hours, $83.3 \%$ of patients in the local group and $93.3 \%$ of patients in the spinal anaesthesia group had score VAS 2 as per visual analogue scale. The two groups are comparable with a p value 0.7326 which is statistically not significant. At four hours post- operative, $53.33 \%$ of patients in the local anaesthesia group and $20 \%$ of patients in the spinal anaesthesia group had VAG score 2 and $30 \%$ patients of the local anaesthesia group and $56.67 \%$ of the spinal anaesthesia group had VAG score of 4 . Again, the two groups are comparable with a p value 0.9097 which is statistically not significant. On post op day 1 , most of the patients in the groups, $46.66 \%$ in local anaesthesia group and $60 \%$ in spinal anaesthesia group had VAG score 2. But none of the patients had severe pain. The two groups are comparable with a $\mathrm{p}$ value of 0.827 showing no significant difference between the two groups.

\begin{tabular}{|c|c|c|}
\hline & Mean time & S.D. \\
\hline Group A & $59.1 \mathrm{~min}$ & $8.76 \mathrm{~min}$ \\
\hline Group B & $40.33 \mathrm{~min}$ & $8.41 \mathrm{~min}$ \\
\hline \multicolumn{3}{|c|}{ Table 1. Table Showing Mean Duration of Surgery } \\
\hline
\end{tabular}

\begin{tabular}{|c|c|c|c|c|c|c|}
\hline $\begin{array}{c}\text { VAG } \\
\text { Score }\end{array}$ & $\begin{array}{c}\text { Group } \\
\text { A }\end{array}$ & $\mathbf{\%}$ & $\begin{array}{c}\text { Group } \\
\text { B }\end{array}$ & $\mathbf{\%}$ & Total & \% \\
\hline 1 & 0 & 0 & 0 & 0 & 0 & 0 \\
\hline 2 & 16 & 53.33 & 6 & 20 & 22 & 36.66 \\
\hline 3 & 0 & 0 & 1 & 3.33 & 1 & 1.66 \\
\hline 4 & 9 & 30 & 17 & 56.66 & 26 & 43.33 \\
\hline 5 & 2 & 6.66 & 1 & 3.33 & 3 & 5 \\
\hline 6 & 3 & 10 & 5 & 16.66 & 8 & 13.33 \\
\hline 7 & 0 & 0 & 0 & 0 & 0 & 0 \\
\hline 8 & 0 & 0 & 0 & 0 & 0 & 0 \\
\hline 9 & 0 & 0 & 0 & 0 & 0 & 0 \\
\hline 10 & 0 & 0 & 0 & 0 & 0 & 0 \\
\hline
\end{tabular}

Table 2. Table Showing Post-Operative Pain at Four Hours 

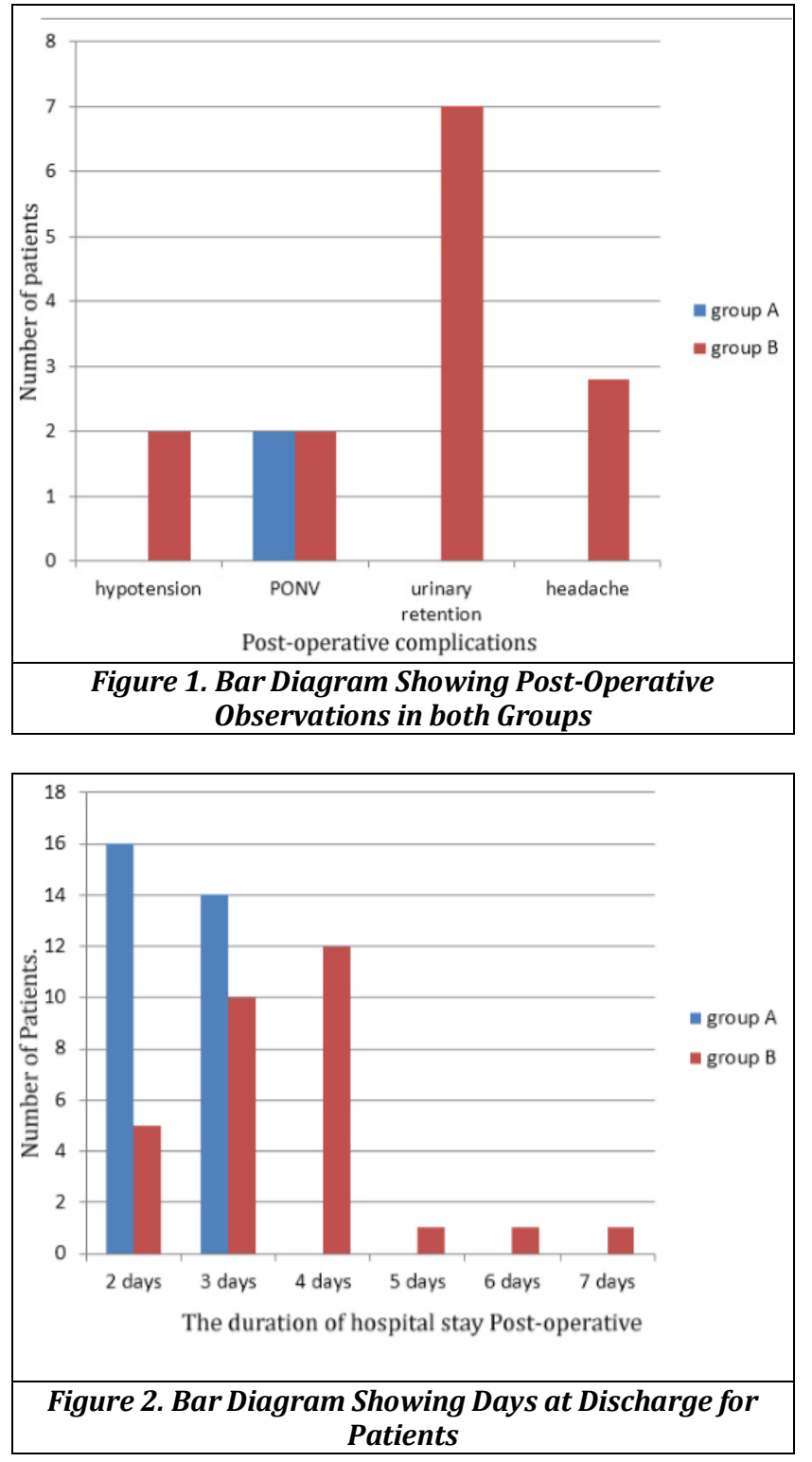

\section{DISCUSSION}

Inguinal hernia repairs are one of the most common surgeries performed throughout the world. Worldwide, some 20 million hernia repairs are accomplished each year. The significance of these large numbers is that, the small variations in practice patterns can have huge socioeconomic implications. Lichtenstein's mesh repair is the time-tested procedure for hernia repair since 25 years and is considered 'the gold-standard' procedure. The present study is a nonrandomized controlled trial study comparing the results of inguinal hernia repair by Lichtenstein meshplasty under local anaesthesia and regional anaesthesia. It includes 60 inguinal hernia repairs, 30 of which are repaired by local anaesthesia administration and considered as 'cases' (Group A) and the remaining 30 repaired by regional anaesthesia and considered as 'controls' (Group B).The incidence of age at presentation of inguinal hernia was maximum between 30-60 years of life. In the present study, the maximum numbers of patients are from $60-70$ and $30-40$ age groups. The mean age of the patients in the present study population is 52.2 years.

The youngest patient in group A was having an age of 21 years and the oldest was having an age of 82 years. The mean age in this group was 53.1 years. The youngest in group B was having an age of 26 years and the oldest person was 76 years old. The mean age in this group was 47.33 years. Similar results were observed in a study conducted by Goel $\mathrm{A}^{12}$ in which mean age of the study population was 59.6 years. In study by Ira in 1987,13 90\% inguinal hernia cases were in males \& $10 \%$ were females. In the study by Lichtenstein in 1988, 94\% were males \& 6\% female patients. At any given age males are more commonly affected then females. In the present study all patients were male.

In both the groups, the incidence of indirect inguinal hernia was more. In the study population, there were 55 cases of indirect inguinal hernia and there were 5 cases of direct inguinal hernia. Among the cases, 43 were right sided and 17 were left sided inguinal hernias. Hence it was observed that right sided inguinal hernia were more common than the left. Right sided inguinal hernia is common type in both direct \& indirect type of hernia. This is due to later descent of right testis \& higher incidence of failure of closure of Processes vaginalis.

The time taken for completion of surgery in group A was $59.1+/-8.76$ minutes and for completion of surgery in group $B$ was $40.33+/-8.41$ minutes. There was no much difference between the time taken for procedure in both the groups. The $\mathrm{p}$ value was $1.61(>0.05)$ and there was no statistically significant difference between the times of surgery between the two groups. Comparable results are obtained in various previous studies Goel A et al 2017), ${ }^{8}$ and Ashok Kumar K et $\operatorname{al}(2013) \cdot{ }^{14}$

In our study both local \& spinal anaesthesia was used in equal number of cases. In the present study none of the patients experienced pain while under spinal anaesthesia. This could be because of higher level of spinal anaesthesia that is $>$ T9 level as attained in present study. Five patients operated under local anaesthesia complained of pain and intravenous midazolam had to be administered to them. The present study can be compared to various previous studies David Y Young which compared the local \& regional anaesthesia for inguinal hernia repair. ${ }^{15}$

In our study people operated under local anaesthesia had significantly over all less complications except for mild pain during surgery. In patients operated under spinal anaesthesia there was significant general complications like intra operative hypotension, postoperative urinary retention \& headache. No patient had any significant blood loss during the surgery. Two patients (6.6\%) experienced hypotension following spinal anaesthesia. No patient in group A had hypotension. The difference is statistically significant with a $p$ value of $0.018(<0.05)$. Two patients each from group A and B experienced post-operative nausea and vomiting which was not severe and resolved with stat dose of anti-emetics. Seven patients in group B experienced urinary retention (23.3\%) whereas none of the patients in group A had urinary retention post operatively. The difference is statistically significant (With a p value of 0.0046). Post-operative headache was seen in $10 \%$ of the group B patients with a significant difference. $(\mathrm{p}=0.025)$ from group $\mathrm{A}$ where no patient had headache. Hence, we can observe that general complications are significantly more in patients who underwent the surgery under spinal anaesthesia. This can be compared with a meta-analysis conducted by Prakash et al (2016) ${ }^{16}$ which included ten original RCT s and showed that patients who underwent the surgery under local anaesthetic 
had significantly less chance for urinary retention and hypotension.

Post-operative pain was analysed using the visual analogue score. The visual analogue score is a pain score which ranges from one to ten in scores. The score can be classified as none (VAS $=0$ ), mild (VAS $=1-3$ ), moderate (VAS= 4-6), severe (VAS $=>7$ ). ${ }^{17}$

Pain was analysed at two hours, four hours and 24 hours post operatively and the response was charted using the visual analogue score. In all the three timings, there was no statistically for 24-hour response, $p$ value 0.909 for the fourhour response and $\mathrm{p}$ value of 0.736 for the two-hour response. Hence, we can observe that there is no statistically significant difference between the two groups in terms of post-operative pain. This when compared to previous studies shows a difference when the patients who underwent the surgery under local anaesthesia had better pain relief post operatively. (Goel A et al 2017).

In addition to the above general complications there were local complications like seroma, hematoma, scrotal oedema, wound infection \& recurrence occurred in both the groups. When compared there was no significant difference between the two groups in the matter of local complications. In present study, the type of anaesthesia had no significant influence on local complications. Only the skills, technique, gentleness \& experience of the surgeons have influence on these complications.

The local complications like seroma, hematoma, and scrotal oedema, taken together from both the groups. One patient from group A developed scrotal oedema (3.3\%) and one patient from group B developed seroma (3.3\%). Both complications treated conservatively with scrotal support \&analgesics and they resolved in 15-20 days. In our no patient developed wound infection following surgery. In previous studies infection occurred in 5.9\% of cases (Maxemo Deysine, 1991), $181.2 \%$ of cases (B Millant 1993),19 \& 2\% cases in (T Faish 2000). ${ }^{20}$ In our present study, no patient developed wound infection. None of the patients had chronic groin pain or paraesthesia.

In our study only post-operative period was calculated, because of delay in pre-operative investigation. In present study $53 \%$ of the LA group discharged by $2^{\text {nd }}$ day \& more than $40 \%$ patients in SA group by 4 th day. Previous studies shows that post-operative stay for short stay surgery was 3-4 days (Sven Kornhale 1976), ${ }^{21} 2.2$ days (Makuria 1979), ${ }^{22} 3.8$ days (S R Canon 1982), ${ }^{23}$ 2-3 days (Glassow 1984). ${ }^{24}$ This is comparable with the previous studies and shows that short stay surgery can very well be practiced in our hospital if local anaesthesia is used for hernia repair.

In the present study the recurrence rate is nil even though it can't be compared because of study group is small \& follow up period was less. It is very difficult to project accurate incidence of recurrence; it will depend on length of follow up. In ideal surgeries the recurrence rate would be $<1 \%$. This is possible only in hernia specialization centers. ${ }^{24}$

In the present study we found that Lichtenstein's hernioplasty under local anaesthesia is safe, simple, effective and economical, with no mortality but had long intraoperative period and more intraoperative pain.

Limitations of the present study are small size, \& inability for $100 \%$ matching between the study groups.

\section{CONCLUSION}

Both local \& spinal anaesthesia can be used for hernia repair on short stay bases, but spinal anaesthesia has higher complication rates compared to local anaesthesia. Local anaesthesia is with less immediate post-operative complication, best suitable for short stay surgery when compared to spinal anaesthesia.

\section{REFERENCES}

[1] Rutkow IM, Robbins AW. Demographic, classificatory and socioeconomic aspects of hernia repair in the United States. Surgical Clinics of North America 1993;73(3):413-26.

[2] Crawford DL, Phillips EH. Laparoscopic and groin hernia surgery. Surgical Clinics of North America 1998;78(6):1047-62.

[3] Chowbey PK, Khullar R, Mehrotra M, et al. Sir Ganga Ram Hospital classification of groin and ventral abdominal wall hernias. Journal of Minimal Access Surgery 2006;2(3):106-9.

[4] Kulacoglu H. Current options in inguinal hernia repair in adult patients. Hippokratia 2011;15(3):223-31.

[5] Lal P. The evolution of surgery for inguinal hernia. Chapter - 9. In: Gupta LR, edr. Recent advances in surgery - 10. Delhi: JayPee Brothers Medical Publishers (P) Ltd., 2006: p. 162-74.

[6] Novik B, Nordin P, Skullman S, et al. More recurrences after hernia mesh fixation with short-term absorbable sutures-a registry study of 82015 Lichtenstein repairs. Arch Surg 2011;146(1):12-7.

[7] Wright AJ, Gardner GC, Fitzgibbons RJ. The Bassini repair and its variants. Chapter - 10. In: Fitzgibbons RJ, Greenburg AG, eds. Nyhus and Condon's hernia. $5^{\text {th }}$ edn. Philadelphia, USA: Lippincott Williams and Wilkins 2002: p. 105-14.

[8] Goel A, Bansal A, Singh A. Comparison of local versus spinal anesthesia in long standing open inguinal hernia repair. Int Surg J 2017;4(11):3701-4.

[9] Kark AE, Kurzer MN, Belsham PA. Three thousand one hundred seventy five primary inguinal hernia repairs advantages of ambulatory open mesh repair using local anaesthesia. J Am Coll Surg 1998;186(4):447-55, discussion 456.

[10] Griffith CA. Repair of inguinal hernia. Chapter - 5. In: Nyhus LM, Coodon RE, eds. Hernia. Philadelphia: JB Lippincott and Co., 1995: p. 106-8.

[11] Calmen CH. Atlas of hernia. Philadelphia: C.V. Mosby Co. Ltd., 1966: p. 1-51.

[12] Rutkow IM. Epidemiologic, economic \& sociologic aspects of hernia surgery in the united states in the 1990's. Surgical Clinics of North America 1998;78(6):941-51, v-vi.

[13] Lichtenstien IL. Herniorraphy: a personal experience with 6,321 cases. American Journal of Surgery 1987;153(6):553-9.

[14] Kumar A, Gothwal S, Sharma S, et al. A prospective comparative study of Lichtenstein tension free hernioplasty under local anesthesia and spinal anesthesia. Scholars Journal of Applied Medical Sciences (SJAMS) Sch J App Med Sci 2013;1(6):934-6. 
[15] Young DV. Comparison of local, spinal \& general anaesthesia for inguinal herniorrhaphy. Am J Surg 1987;153(6):560-3.

[16] Prakash D, Heskin L, Doherty S, et al. Local anaesthesia versus spinal anaesthesia in inguinal hernia repair: a systematic review and meta-analysis. The Surgeon 2017;15(1)47-57.

[17] Wantz GE. Complications of inguinal hernia repair. Surgical Clinics of North America 1984;64(2):287-98.

[18] Deysine M, Grimson RC, Soroff HS. Inguinal herniorrhaphy. Reduced morbidity by service standardization. Archive of surgery 1991;126(5):62830.

[19] Millat B, Fingerhut A, Gignoux M, et al. Factors associated with early discharge after inguinal hernia repair in 500 consecutive unselected patients. French Associations for Surgical Research. British Journal of Surgery 1993;80(9):1158-60.
[20] Fasih T, Mahapatra TK, Waddington RT. Early results of inguinal hernia repair by 'mesh plug' technique, first 200 cases. Annals of the Royal College of Surgery Engl 2000;82(6):396-400.

[21] Hall SK, Molsson A. Ambulatory inguinal hernia repair compared with short stay surgery. American Journal of Surgery 1982;132:32-3.

[22] Makuri T, Alexander-Williams T, Keighley MR. Comparison between general \& local anaesthesia for repair of groin hernias. Annals of Royal College Surgery England 1979;61(4):291-4.

[23] Canon SR, Ralphs DN, Bolton JP, et al. Early discharge following hernia repair in unselected patients. British Journal of Surgery 1982;69(2):112-3.

[24] Glassow F. Inguinal hernia repair using local anaesthesia. Annals of the Royal College of Surgeons of England 1984;66(6):382-7. 